\title{
ON AN INVERSE PROBLEM FOR ANISOTROPIC CONDUCTIVITY IN THE PLANE
}

\author{
GENNADI HENKIN AND MATTEO SANTACESARIA
}

\begin{abstract}
Let $\hat{\Omega} \subset \mathbb{R}^{2}$ be a bounded domain with smooth boundary and $\hat{\sigma}$ a smooth anisotropic conductivity on $\hat{\Omega}$. Starting from the Dirichlet-to-Neumann operator $\Lambda_{\hat{\sigma}}$ on $\partial \hat{\Omega}$, we give an explicit procedure to find a unique (up to a biholomorphism) domain $\Omega$, an isotropic conductivity $\sigma$ on $\Omega$ and the boundary values of a quasiconformal diffeomorphism $F: \hat{\Omega} \rightarrow \Omega$ which transforms $\hat{\sigma}$ into $\sigma$.
\end{abstract}

\section{INTRODUCTION}

Let $\Omega \subset \mathbb{R}^{2}$ be a bounded domain, and let $\sigma$ be a $C^{2}$-anisotropic conductivity defined over $\Omega$, i.e. $\sigma=\left(\sigma^{i j}\right)$ is a positive definite symmetric matrix on $\bar{\Omega}$ in the $C^{2}$ class. The corresponding Dirichlet-to-Neumann map is the operator $\Lambda_{\sigma}: C^{1}(\partial \Omega) \rightarrow L^{p}(\partial \Omega), p<\infty$ defined by

$$
\Lambda_{\sigma} f=\left.\sigma \frac{\partial u}{\partial \nu}\right|_{\partial \Omega}
$$

where $f \in C^{1}(\partial \Omega), \nu$ is the outer normal of $\partial \Omega$, and $u$ is the $C^{1}(\bar{\Omega})$-solution of the Dirichlet problem

$$
\nabla \cdot(\sigma \nabla u)=0 \text { on } \Omega,\left.\quad u\right|_{\partial \Omega}=f .
$$

The equation (1.2) represents the conservation of the electrical charge on $\Omega$ if the voltage potential $f$ is applied to $\partial \Omega$, and $\Lambda_{\sigma} f$ is the current flux at the boundary. The following inverse problem arises from this construction: how much information about $\sigma$ can be detected from the knowledge of the mapping $\Lambda_{\sigma}$ ?

Inverse boundary values problems of such a type were formulated in precise mathematical terms by I. Gel'fand [10] and by A. Calderon [5]. These problems arise naturally in several areas: geophysical electrical prospecting (L. Slichter [17], V. Druskin [6]), medical imaging (D. Barber, B. Brown [3]), nondestructive testing of materials (A. Friedman, M. Vogelius [9]), etc.

It is not possible to determine $\sigma$ uniquely from $\Lambda_{\sigma}$. This was discovered by L. Tartar (see [12]). Indeed, let $\Phi: \bar{\Omega} \rightarrow \bar{\Omega}$ be a diffeomorphism with

1991 Mathematics Subject Classification. Primary 35R30; Secondary 32G05.

Key words and phrases. Inverse conductivity problem; Anisotropic conductivity; Deformations of complex structures. 
$\left.\Phi\right|_{\partial \Omega}=\mathrm{Id}$, where Id is the identity map. Then we can define the pushforward of $\sigma$ as

$$
\Phi_{*} \sigma=\left(\frac{{ }^{t}(D \Phi) \sigma(D \Phi)}{|\operatorname{det}(D \Phi)|}\right) \circ \Phi^{-1},
$$

where $D \Phi$ is the matrix differential of $\Phi$, and one verifies that $\Lambda_{\Phi_{*} \sigma}=\Lambda_{\sigma}$. In dimension two this is the only obstruction to unique identifiability of the conductivity. The anisotropic problem can be reduced to the isotropic one by using isothermal coordinates (Sylvester [18]), and combining this technique with the result of Nachman for isotropic conductivities ([15]) we obtain the uniqueness result for anisotropic conductivities with two derivatives. The optimal regularity condition was later obtained by Astala-Lassas-Paivarinta, who proved the uniqueness for $L^{\infty}$-conductivities in [2]: for an anisotropic conductivity $\sigma \in L^{\infty}(\Omega)\left(\Omega \subset \mathbb{R}^{2}\right.$ bounded simply connected domain) the Dirichlet-to-Neumann map determines the equivalence class of conductivities $\sigma^{\prime}$ such that there exists a diffeomorphism $\Phi: \Omega \rightarrow \Omega$ in the $W^{1,2}$ class with $\left.\Phi\right|_{\partial \Omega}=\mathrm{Id}$ and $\sigma^{\prime}=\Phi_{*} \sigma$.

The main purpose of this article is to clarify and show what one can explicitly reconstruct from a given Dirichlet-to-Neumann operator in the anisotropic case. From the results obtained in [18], [16], [15], [11] we have deduced

Theorem 1. Let $\hat{\Omega} \subset \mathbb{R}^{2}$ be a bounded domain with $C^{1}$ boundary and let $\hat{\sigma}$ be a $C^{2}$-anisotropic conductivity on $\hat{\Omega}$, isotropic in a neighbourhood of $\partial \hat{\Omega}$. Suppose we know $\Lambda_{\hat{\sigma}}: C^{1}(\partial \hat{\Omega}) \rightarrow L^{p}(\partial \hat{\Omega}), p<\infty$.

Then we can reconstruct a unique domain $\Omega \subset \mathbb{R}^{2} \sim \mathbb{C}$ (up to a biholomorphism), an isotropic conductivity $\sigma$ on $\Omega$ and the boundary values $\left.F\right|_{\partial \hat{\Omega}}$ of a quasiconformal $C^{1}$-diffeomorphism $F: \hat{\Omega} \rightarrow \Omega$ such that $\sigma=F_{*} \hat{\sigma}$.

The new point in this statement is the existence of $F: \hat{\Omega} \rightarrow \Omega$ (and its explicit reconstruction at the boundary) without any assumption on the topology of $\hat{\Omega}$. Early in [2] this result was proved for simply connected domains, a situation in which the question about deformations of complex structures of $\hat{\Omega}$ does not make sense.

Our main tool, as in [18] and [2, is the global solution $F$ of a certain Beltrami equation equipped with an asymptotic condition, which takes our anisotropic conductivity $\hat{\sigma}$ into an isotropic one, $\sigma$, defined in general over a different domain $\Omega=F(\hat{\Omega})$. With the help of $F$ we then show the existence and uniqueness of a family of solutions $\hat{\psi}(z, \lambda)$ of the anisotropic conductivity equation, with special asymptotics at infinity, using also the existence of such type of functions in the isotropic case, that we call $\psi(w, \lambda)$ (firstly introduced by Faddeev in [7]; see [16], 15] for the main properties). Then we show how 
one can reconstruct the boundary values of $\hat{\psi}$ from the Dirichlet-to-Neumann operator $\Lambda_{\hat{\sigma}}$, for any $\lambda$, with a Fredholm-type integral equation, following the work of Gutarts ([1]). This is a generalization of R. Novikov's method for isotropic conductivities ([16]). We also show how to find the boundary values of $F$ from the knowledge of $\left.\hat{\psi}\right|_{\partial \hat{\Omega}}$ (generalizing the result in [18] and [2]), and so we find $F(\partial \hat{\Omega})=\partial \Omega$ (therefore also $\Omega$ ).

After this, we explain how the knowledge of $\Lambda_{\hat{\sigma}},\left.\hat{\psi}\right|_{\partial \hat{\Omega}}$ and $\left.F\right|_{\partial \hat{\Omega}}$ suffices to reconstruct the isotropic scattering amplitude $b(\lambda)$. We give also another method: we define the anisotropic scattering amplitude $\hat{b}(\lambda)$, and we show that it is equal to the isotropic one, proving that it is essentially a quasiconformal invariant. This result was already included in [11]; here we give a new simpler proof.

Thus with both methods, starting from $\left.\hat{\psi}\right|_{\partial \hat{\Omega}}$ we can reconstruct the isotropic scattering amplitude: this allows us to write the $\bar{\partial}$-equation which will permit us to find the isotropic conductivity $\sigma$ on $\Omega$, by the NovikovNachman reconstruction scheme ([16, [15]).

Our scheme can be summarized in the following diagram

$$
\left.\Lambda_{\hat{\sigma}} \rightarrow \hat{\psi}\right|_{\partial \hat{\Omega}} \rightarrow\left\{\begin{array} { c } 
{ b ( \lambda ) } \\
{ F | _ { \partial \hat { \Omega } } }
\end{array} \rightarrow \left\{\begin{array}{c}
\sigma \\
\Omega
\end{array}\right.\right.
$$

All steps of this reconstruction scheme are explicit and can be numerically implemented using the Novikov-Nachman reconstruction-type algorithm [16], [15]. Therefore, our paper admits potential practical applications.

Remark 1. Although we cannot reconstruct $\hat{\sigma}$ uniquely, for the applications it may be useful to find one representative of the equivalence class of $\hat{\sigma}$. To do this, using our theorem it suffices to find a diffeomorphism $G: \hat{\Omega} \rightarrow \Omega$ with fixed boundary values (which are the boundary values of a quasiconformal mapping, in our notation $\left.F\right|_{\partial \hat{\Omega}}$ ), and no other particular restriction: in this way $\left(G^{-1}\right)_{*} \sigma$ will be a representative of $\hat{\sigma}$. If $\Omega$ is simply connected one can use the Ahlfors-Beurling extension theorem for quasi-symmetric homeomorphism of the circle ([1, Thm. 2, p.69]).

Remark 2. An analogous result to our Theorem 1 is valid also on bordered surfaces in $\mathbb{R}^{3}$.

Remark 3. One of the referees has drown our attention to the possible relation of our paper to the publications [13] and [14]. In these papers is shown that for the inverse isotropic-conductivity problem in an inaccurately modelled (simply connected) domain there is a unique anisotropic conductivity, corresponding to the boundary measurements, which has the minimal possible anisotropy; this minimally-anisotropic conductivity can be «isotropized», 
using Beltrami equation, in order to obtain the original isotropic conductivity (up to biholomorphisms of simply connected domains). These papers have certainly some common parts with [2, where the inverse anisotropicconductivity problem on simply connected domains is studied. But these publications have no common points with our paper; indeed our main novelty consists in the complete study of the inverse anisotropic-conductivity problem in arbitrary domains (not necessarily simply connected) with smooth boundaries. Nevertheless, our results can be applied to extend the abovementioned publications to the case of non simply connected domains.

\section{The Beltrami equation and Faddeev-type anisotropic} SOLUTIONS

We identify $\mathbb{R}^{2}$ with $\mathbb{C}$ by the map $(x, y) \mapsto x+i y=z$ and we use the notation

$$
\partial_{z}=\frac{1}{2}\left(\partial_{x}-i \partial_{y}\right), \quad \partial_{\bar{z}}=\frac{1}{2}\left(\partial_{x}+i \partial_{y}\right)
$$

where $\partial_{x}=\partial / \partial x$ and $\partial_{y}=\partial / \partial y$. We will also use the differential operators $\partial, \bar{\partial}$ such that $\partial f=\partial_{z} f d z, \bar{\partial} f=\partial_{\bar{z}} f d \bar{z}$, with $d z=d x+i d y, d \bar{z}=d x-i d y$. We also recall the identity $d=\partial+\bar{\partial}$.

We can suppose that $\hat{\sigma}$, already isotropic near $\partial \hat{\Omega}$, is the identity near $\partial \hat{\Omega}$ (see [15] for the reduction to this case). Besides, we extend $\hat{\sigma}$ to the whole complex plane by putting $\hat{\sigma}=I$ for $z \in \mathbb{C} \backslash \hat{\Omega}$. Then, for the conductivity $\hat{\sigma}=\hat{\sigma}^{i j}$ we define the following Beltrami coefficient

$$
\mu_{1}(z)=\frac{-\hat{\sigma}^{11}(z)+\hat{\sigma}^{22}(z)-2 i \hat{\sigma}^{12}(z)}{\hat{\sigma}^{11}(z)+\hat{\sigma}^{22}(z)+2 \sqrt{\operatorname{det}(\hat{\sigma})}}
$$

which satisfies $\left|\mu_{1}(z)\right| \leq k<1$ and is compactly supported in $\hat{\Omega}$. We now recall the existence of a diffeomorphism that transforms $\hat{\sigma}$ into an isotropic conductivity.

Proposition 2. (Sylvester [18, Prop. 2.1]) There is a quasiconformal $C^{1}$ diffeomorphism

$F: \mathbb{C} \rightarrow \mathbb{C}$ such that

$$
F(z)=z+O\left(\frac{1}{z}\right) \quad \text { as }|z| \rightarrow \infty
$$

and for which

$$
\left(F_{*} \hat{\sigma}\right)(z)=\sigma(z) I:=(\operatorname{det}(\hat{\sigma}))^{1 / 2} \circ F^{-1}(z) I .
$$

Thanks to results by Ahlfors and Vekua ([1], [19]), $F$ is obtained as the solution of the Beltrami equation $\partial_{\bar{z}} F=\mu_{1} \partial_{z} F$, so $F$ is holomorphic in $\mathbb{C} \backslash \hat{\Omega}$. 
Proposition 3. There exist unique Faddeev-type solutions of the anisotropic conductivity equation, i.e. functions $\hat{\psi}(z, \lambda)$ such that

$$
\nabla \cdot(\hat{\sigma}(\nabla \hat{\psi}))=0
$$

for all $z \in \mathbb{C}, \quad \lambda \in \mathbb{C}$, and $\hat{\psi}(z, \lambda)=e^{\lambda z}\left(1+O\left(\frac{1}{z}\right)\right)$ when $z \rightarrow \infty$.

Proposition 3 for the case det $\hat{\sigma}$ close to a constant was obtained firstly in [18.

Proof. We define $\Omega=F(\hat{\Omega})$ and $q=\frac{\Delta \sigma^{1 / 2}}{\sigma^{1 / 2}}$. It is known that if $u$ is a solution of $\nabla \cdot(\sigma \nabla u)=0$ in $\Omega$, then $\tilde{u}=\sigma^{1 / 2} u$ is a solution of

$$
-\Delta \tilde{u}+q \tilde{u}=0
$$

in $\Omega$. From [4], [15] and [16], we have that for every $\lambda \in \mathbb{C}$ there is a unique solution $\tilde{\psi}(w, \lambda)$ of (2.2) with the asymptotic behaviour $\tilde{\psi}(w, \lambda)=e^{\lambda w}(1+$ $\left.O\left(\frac{1}{w}\right)\right)$ when $w \rightarrow \infty$. So we directly have that $\psi(w, \lambda):=\sigma^{-1 / 2} \tilde{\psi}(w, \lambda)$ is a solution of $\nabla \cdot(\sigma \nabla \psi)=0$ with the same asymptotic (because $\sigma=1$ outside $\Omega)$.

Now let $\hat{\psi}(z, \lambda)$ be a Faddeev-type anisotropic solution. If we consider $\psi^{\prime}(w, \lambda)=\hat{\psi}\left(F^{-1}(w), \lambda\right)$, we have that $\nabla \cdot\left(\sigma \nabla \psi^{\prime}\right)=0$ from the construction of $\sigma$. Using the properties of $F$ and $\hat{\psi}$, we get, for $w \rightarrow \infty$,

$$
\begin{aligned}
\psi^{\prime}(w, \lambda)=\hat{\psi}\left(F^{-1}(w), \lambda\right) & =e^{\lambda F^{-1}(w)}\left(1+O\left(\frac{1}{\left|F^{-1}(w)\right|}\right)\right) \\
& =e^{\lambda w}\left(1+O\left(\frac{1}{1+|w|}\right)\right)
\end{aligned}
$$

showing that $\psi^{\prime}(w, \lambda)$ satisfies the same asymptotic of $\psi(w, \lambda)$. From the uniqueness of $\psi(w, \lambda)$ we obtain

$$
\hat{\psi}(z, \lambda)=\psi(F(z), \lambda)
$$

which proves both existence and uniqueness.

From the equality (2.3) we can also derive a useful formula to calculate $\left.F\right|_{\partial \hat{\Omega}}$. In fact, results in [8] also indicate how the family of Faddeev-type solutions behaves with respect to $\lambda$. We have indeed $\left|e^{-w \lambda} \tilde{\psi}(w, \lambda)-1\right| \rightarrow 0$ as $|\lambda| \rightarrow \infty$ for every fixed $w \in \mathbb{C}$. If we take $w \in \mathbb{C} \backslash \Omega$ the same limit is also valid for $\psi(w, \lambda)$; combining this with (2.3) we deduce the following formula.

Proposition 4. ([18, Prop. 2.7]) For all $z \in \mathbb{C} \backslash \hat{\Omega}$ (in particular for $z \in \partial \hat{\Omega}$ ) we have

$$
\lim _{|\lambda| \rightarrow \infty} \frac{\log (\hat{\psi}(z, \lambda))}{\lambda}=\lim _{|\lambda| \rightarrow \infty} \frac{\log (\psi(F(z), \lambda))}{\lambda}=F(z)
$$




\section{An integral EQUATION FOR $\left.\hat{\psi}\right|_{\partial \hat{\Omega}}$}

Following the approach of [11, we show that, as in the isotropic case, we can find $\left.\hat{\psi}\right|_{\partial \hat{\Omega}}$ through a Fredholm-type integral equation.

The main idea is to decompose the differential operator $-\nabla \cdot \hat{\sigma} \nabla$ as $-\Delta+M$, where $M$ is a compactly supported operator. So we can characterize $\hat{\psi}(z, \lambda)$ as the solution of the following integral equation:

$$
\hat{\psi}(z, \lambda)=e^{z \lambda}-\frac{i}{2} \int_{\hat{\Omega}} G(z-w, \lambda) M \hat{\psi}(w, \lambda) d w \wedge d \bar{w},
$$

where

$$
G(z, \lambda)=\frac{i e^{\lambda z}}{2(2 \pi)^{2}} \int_{\mathbb{C}} \frac{e^{i(w \bar{z}+\bar{w} z)} d w \wedge d \bar{w}}{w(\bar{w}-i \lambda)}, \quad z \in \mathbb{C}, \lambda \in \mathbb{C}
$$

is the Faddeev-Green function for the Laplacian.

Proposition 5. (111, Lemma 2.4]) For every $\lambda \in \mathbb{C}$ the boundary value of $\hat{\psi}$ satisfies

$$
\left.\hat{\psi}(z, \lambda)\right|_{\partial \hat{\Omega}}=e^{z \lambda}-\int_{\partial \hat{\Omega}} G(z-w, \lambda)\left(\Lambda_{\hat{\sigma}}-\Lambda_{0}\right) \hat{\psi}(w, \lambda) d w,
$$

where $\Lambda_{0}$ is the Dirichlet-to-Neumann operator of the standard Laplacian (or for the case of constant conductivity).

This follows from the identity

$$
\int_{\partial \hat{\Omega}} u_{0}\left(\Lambda_{\hat{\sigma}}-\Lambda_{0}\right) u=\int_{\hat{\Omega}} u_{0} M u
$$

where $u_{0}, u \in W^{1,2}(\hat{\Omega}), \nabla \cdot(\hat{\sigma} \nabla u)=0, \Delta u_{0}=0$ in $\hat{\Omega}$.

The fact that the integral equation (3.1) is of Fredholm type in the Sobolev space $W^{s, 2}(\partial \hat{\Omega})$ is the content of [11, Lemma 2.5], and it is uniquely solvable by [11, Lemma 2.6] (these properties are implied by the same results in the isotropic case [15]).

\section{Reconstruction of the SCATtering Amplitude}

Following [8], we define the non-physical scattering amplitude for the isotropic inverse problem as

$$
b(\lambda)=\int_{\Omega} e^{-\bar{\lambda} \bar{w}} q(w) \tilde{\psi}(w, \lambda) d w .
$$

From [16] we have

$$
b(\lambda)=\int_{\partial \Omega} e^{-\bar{\lambda} \bar{w}}\left(\Lambda_{q}-\Lambda_{0}\right) \tilde{\psi}(w, \lambda) d w
$$

where $\Lambda_{q}$ is the Dirichlet-to-Neumann operator of the Schrödinger equation (2.2). 
Since $\sigma$ is the identity near $\partial \Omega$, equation $\Lambda_{q}=\sigma^{-1 / 2}\left(\Lambda_{\sigma}+\frac{1}{2} \frac{\partial \sigma}{\partial \nu}\right) \sigma^{-1 / 2}$ reads $\Lambda_{q}=\Lambda_{\sigma}$, and $\left.\tilde{\psi}\right|_{\partial \Omega}=\left.\psi\right|_{\partial \Omega}$, so

$$
b(\lambda)=\int_{\partial \Omega} e^{-\bar{\lambda} \bar{w}}\left(\Lambda_{\sigma}-\Lambda_{0}\right) \psi(w, \lambda) d w .
$$

Thus, for the reconstruction of $b$, it is sufficient to determine $\Lambda_{\sigma}$ and $\left.\psi\right|_{\partial \Omega}$. By (2.3) we already know $\left.\psi\right|_{\partial \Omega}$; for the determination of $\Lambda_{\sigma}$, by arguments of [2], we obtain the identity

$$
\int_{\partial \hat{\Omega}} \hat{u} \Lambda_{\hat{\sigma}} \hat{v}=\int_{\partial \Omega} u \Lambda_{\sigma} v
$$

which holds for any $\hat{u}, \hat{v} \in C^{1}(\partial \hat{\Omega})$ and $u, v \in C^{1}(\partial \Omega)$ such that $\hat{u}=u \circ F$ and $\hat{v}=v \circ F$ (this follows directly from the properties of $F$ and the symmetry of the two Dirichlet-to-Neumann operators). So we find $\Lambda_{\sigma}$ from $\Lambda_{\hat{\sigma}}$ and $\left.F\right|_{\partial \hat{\Omega}}$.

4.1. Complementary result. We give here another method to find $b(\lambda)$. Inspired by [11, we define the anisotropic scattering amplitude as

$$
\hat{b}(\lambda)=\int_{\hat{\Omega}} e^{-\bar{\lambda} \bar{z}} M \hat{\psi}(z, \lambda) d z
$$

and we have the following result.

Proposition 6. $b(\lambda)=\hat{b}(\lambda)$

We will need the following lemma

Lemma 7. For every $\phi \in C^{1}(\partial \hat{\Omega}), \psi \in C^{1}(\hat{\Omega})$ solution of $\nabla \cdot(\hat{\sigma} \nabla \psi)=$ $(\Delta-M) \psi=0$ in $\hat{\Omega}$, we have

$$
\int_{\partial \hat{\Omega}} \phi\left(\Lambda_{\hat{\sigma}}-\Lambda_{0}\right) \psi=2 i \int_{\partial \hat{\Omega}} \phi\left(\bar{\partial} \psi-\bar{\partial} \psi_{0}\right)
$$

where $\Delta \psi_{0}=0$ in $\hat{\Omega}$ and $\left.\psi_{0}\right|_{\partial \hat{\Omega}}=\left.\psi\right|_{\partial \hat{\Omega}}$.

Proof. Let $a \in C^{1}(\hat{\Omega})$ such that $\left.a\right|_{\partial \hat{\Omega}}=\phi$, and $w=x+i y$. From the definition of the Dirichlet-to-Neumann operator and from Stokes' theorem, one has

$$
\int_{\partial \hat{\Omega}} \phi\left(\Lambda_{\hat{\sigma}}-\Lambda_{0}\right) \psi=\int_{\hat{\Omega}}\left(\nabla a \cdot \nabla\left(\psi-\psi_{0}\right)+a M \psi\right) d x d y
$$

and by Stokes' theorem and by the identity $\Delta=4 \frac{\partial^{2}}{\partial z \partial \bar{z}}$

$$
2 i \int_{\partial \hat{\Omega}} \phi\left(\bar{\partial} \psi-\bar{\partial} \psi_{0}\right)=2 i \int_{\hat{\Omega}} \partial a \wedge\left(\bar{\partial} \psi-\bar{\partial} \psi_{0}\right)+\int_{\hat{\Omega}} a M \psi d x d y .
$$


Writing in coordinates we get

$$
\partial a \wedge \bar{\partial}\left(\psi-\psi_{0}\right)=\frac{1}{2 i} \nabla a \cdot \nabla\left(\psi-\psi_{0}\right) d x d y+\frac{1}{2} d a \wedge d\left(\psi-\psi_{0}\right) .
$$

Again by Stokes' thorem we have

$$
\int_{\hat{\Omega}} d a \wedge d\left(\psi-\psi_{0}\right)=-\int_{\partial \hat{\Omega}}\left(\psi-\psi_{0}\right) d a=0
$$

because $\left.\psi\right|_{\partial \hat{\Omega}}=\left.\psi_{0}\right|_{\partial \hat{\Omega}}$. The proof follows.

Proof of Proposition [6. From identity (3.2) we find

$$
\hat{b}(\lambda)=\int_{\partial \hat{\Omega}} e^{-\bar{\lambda} \bar{z}}\left(\Lambda_{\hat{\sigma}}-\Lambda_{0}\right) \hat{\psi}(z, \lambda) d z .
$$

Using the lemma we find

$$
\begin{aligned}
& \hat{b}(\lambda)=2 i \int_{\partial \hat{\Omega}} e^{-\bar{\lambda} \bar{z}}\left(\bar{\partial} \hat{\psi}-\bar{\partial} \hat{\psi}_{0}\right)=2 i \int_{\partial \hat{\Omega}} e^{-\bar{\lambda} \bar{z}} \hat{\psi} \hat{\psi} \\
& b(\lambda)=2 i \int_{\partial \Omega} e^{-\bar{\lambda} \bar{w}}\left(\bar{\partial} \psi-\bar{\partial} \psi_{0}\right)=2 i \int_{\partial \Omega} e^{-\bar{\lambda} \bar{w}} \bar{\partial} \psi
\end{aligned}
$$

where the second equalities follows from Stokes' theorem, the fact that $e^{-\bar{\lambda} \bar{z}}$ (resp. $e^{-\bar{\lambda} \bar{w}}$ ) is antiholomorphic and $\hat{\psi}_{0}\left(\right.$ resp. $\left.\psi_{0}\right)$ is harmonic in $\hat{\Omega}$ (resp. in $\Omega$ ).

If we call $z=G(w)=F^{-1}(w)$ we find, from (4.6),

$$
\begin{aligned}
\hat{b}(\lambda) & =2 i \int_{\partial \hat{\Omega}} e^{-\overline{\lambda \bar{z}} \frac{\partial \hat{\psi}}{\partial \bar{z}}} d \bar{z} \\
& =2 i \int_{\partial \Omega} e^{-\overline{\lambda G(w)}} \overline{\left(\frac{\partial F}{\partial z}\right)} \frac{\partial \psi}{\partial \bar{w}}(w, \lambda) \overline{\left(\frac{\partial G}{\partial w}\right)} d \bar{w} \\
& =2 i \int_{\partial \Omega} e^{-\overline{\lambda G(w)}} \bar{\partial} \psi
\end{aligned}
$$

because $F$ (resp. $G$ ) is holomorphic in a neighbourhood of $\partial \hat{\Omega}$ (resp. $\partial \Omega$ ), and from the equality $\psi \circ F=\hat{\psi}$.

To see that (4.8) is equal to (4.7) we proceed as follows. Let $\Omega_{R}=\{z \in$ $\mathbb{C}:|z|<R\}$ the disk of radius $R$, and let $R$ be sufficiently large to have $\bar{\Omega} \subset \Omega_{R}$. We apply Stokes' theorem to $\Omega_{R} \backslash \Omega$ and we obtain, for every quasiconformal homeomorphism $E: \mathbb{C} \rightarrow \mathbb{C}$, holomorphic in $\mathbb{C} \backslash \Omega$,

$$
\int_{\partial \Omega} e^{-\overline{\lambda E(w)}} \bar{\partial} \psi=\int_{\partial \Omega_{R}} e^{-\overline{\lambda E(w)}} \bar{\partial} \psi+\int_{\Omega_{R} \backslash \Omega} \partial\left(e^{-\overline{\lambda E(w)}} \bar{\partial} \psi\right)
$$

but the last term vanishes, because $e^{-\overline{\lambda E(w)}}$ is anti-holomorphic and $\partial \bar{\partial} \psi=0$ in $\mathbb{C} \backslash \Omega$. 
So the identity

$$
\int_{\partial \Omega} e^{-\overline{\lambda E(w)}} \bar{\partial} \psi=\int_{\partial \Omega_{R}} e^{-\overline{\lambda E(w)}} \bar{\partial} \psi
$$

is true for $R \gg 0, E(w)=G(w)$ and $E(w)=w$. As we have $G(w)=$ $w+O\left(\frac{1}{|w|}\right)$ for $w \rightarrow \infty$, using the lemma we deduce

$$
\begin{aligned}
\hat{b}(\lambda) & =2 i \int_{\partial \Omega} e^{-\overline{\lambda G(w)}} \bar{\partial} \psi=\lim _{R \rightarrow \infty} 2 i \int_{\partial \Omega_{R}} e^{-\overline{\lambda G(w)}} \bar{\partial} \psi \\
& =\lim _{R \rightarrow \infty} 2 i \int_{\partial \Omega_{R}} e^{-\overline{\lambda w}} \bar{\partial} \psi=2 i \int_{\partial \Omega} e^{-\overline{\lambda w}} \bar{\partial} \psi=b(\lambda)
\end{aligned}
$$

\section{The $\bar{\partial}$-EQUATION AND THE RECONSTRUCTION OF $\sigma$}

Here we follow the steps of [16] to reconstruct isotropic conductivities. The function $\mu(w, \lambda)=\tilde{\psi}(w, \lambda) e^{-\lambda w}$ satisfies the following $\bar{\partial}$-equation with respect to $\lambda$

$$
\frac{\partial \mu(w, \lambda)}{\partial \bar{\lambda}}=\frac{b(\lambda)}{4 \pi \bar{\lambda}} e^{\bar{\lambda} \bar{w}-\lambda w} \overline{\mu(w, \lambda)}
$$

This is equivalent to the integral equation:

$$
\mu(w, \lambda)=1+\frac{1}{8 \pi^{2} i} \int_{\mathbb{C}} \frac{b\left(\lambda^{\prime}\right)}{\left(\lambda^{\prime}-\lambda\right) \bar{\lambda}^{\prime}} e^{\bar{\lambda}^{\prime} \bar{w}-\lambda^{\prime} w} \overline{\mu\left(w, \lambda^{\prime}\right)} d \lambda^{\prime} \wedge d \bar{\lambda}^{\prime}
$$

because $\mu \rightarrow 1$ when $w \rightarrow \infty$. By results of [15], equation (5.2) is solvable, and one can find $\sigma(w)$ from the integral formula

$$
\sigma^{1 / 2}(w)=\mu(w, 0)=1+\frac{1}{8 \pi^{2} i} \int_{\mathbb{C}} \frac{b(\lambda)}{|\lambda|^{2}} e^{\bar{\lambda} \bar{w}-\lambda w} \overline{\mu(w, \lambda)} d \lambda \wedge d \bar{\lambda}, \quad \forall w \in \mathbb{C}
$$

or from the more stable general formula

$$
\frac{\Delta \sigma^{1 / 2}(w)}{\sigma^{1 / 2}(w)}=\frac{\Delta \tilde{\psi}(w, \lambda)}{\tilde{\psi}(w, \lambda)}, \forall w \in \mathbb{C}, \forall \lambda \in \mathbb{C} .
$$

\section{REFERENCES}

[1] Ahlfors, L. V., Lectures On Quasiconformal Mappings, D. Van Nostrand Company, Inc. 1966.

[2] Astala, K., Lassas, M., Päivärinta, L., Calderón's inverse problem for anisotropic conductivity in the plane, Commun. Partial Differ. Equ. 30, 2005, 207-224.

[3] Barber, D. C., Brown, B. H., Applied potential tomography J. Phys. E: Sci. Instrum. 17, 1984, 723-733.

[4] Beals, R., Coifman, R., The spectral problem for the Davey-Stewartson and Ishimori hierarchies, In: "Nonlinear Evolution Equations : Integrability and Spectral Methodes", Proc. Workshop, Como, Italy 1988, Proc. Nonlinear Sci., 1990, 15-23. 
[5] Calderón, A.P., On an inverse boundary problem, Seminar on Numerical Analysis and its Applications to Continuum Physics, Soc. Brasiliera de Matematica, Rio de Janeiro, 1980, 61-73.

[6] Druskin, V., The unique solution of the inverse problem in electrical surveying and electrical well logging for piecewise-constant conductivity, Physics of the Solid Earth, 18, 1982, 51-53.

[7] Faddeev, L. D., Growing solutions of the Schrödinger equation, Dokl. Akad. Nauk SSSR 165, No. 3, 1965, 514-517.

[8] Faddeev, L. D., The inverse problem in the quantum theory of scattering. II, Current Problems in Mathematics [in Russian], Vol. 3, Akad. Nauk SSSR, Vsesoyuznyi Inst. Nauchnoi i Tekhnicheskoi Informatsii, Moscow, 1974, 93-180.

[9] Friedman, A., Vogelius, M., Identification of small inhomogeneities of extreme conductivity by boundary measurements: a theorem on continuous dependence, Arch. Rational Mech. Anal. 105, no. 4, 1989, 299-326.

[10] Gel'fand, I.M., Some problems of functional analysis and algebra, Proc. Int. Congr. Math., Amsterdam, 1954, 253-276.

[11] Gutarts, B., The inverse boundary problem for the two-dimensional elliptic equation in anisotropic media, J. Math. Stat. Allied Fields 1, 2007.

[12] Kohn, R., Vogelius, M., Determining conductivity by boundary measurements II. Interior Results, Comm. Pure Appl. Math. 38, 1985, 643-667.

[13] Kolehmainen, V., Lassas, M., Ola, P., The inverse conductivity problem with an imperfectly known boundary, SIAM J. Appl. Math. 66, no. 2, 2005, 365-383.

[14] Kolehmainen, V., Lassas, M., Ola, P., Calderón's Inverse Problem with an Imperfectly Known Boundary and Reconstruction Up to a Conformal Deformation, SIAM J. Math. Anal. 42, no. 3, 2010, 1371-1381

[15] Nachman, A., Global uniqueness for a two-dimensional inverse boundary value problem, Ann. Math. 143, 1996, 71-96.

[16] Novikov, R., Multidimensional inverse spectral problem for the equation $-\Delta \psi+(v-$ $E u) \psi=0$, Funct. Anal. and Appl. 22, 1988, 263-272.

[17] Slichter, L., An inverse boundary value problem in electrodynamics, Physics 4, 1933, 411-418.

[18] Sylvester, J., An Anisotropic Inverse Boundary Value Problem, Comm. Pure Appl. Math 43, 1990, 201-32.

[19] Vekua, I. N., Generalized Analytic Functions, Pergamon Press Ltd. 1962.

(G. Henkin) Université Pierre et Marie Curie, case 247, 4, Place Jussieu, 75252, Paris, France

E-mail address: henkin@math.jussieu.fr

(M. Santacesaria) Centre de Mathématiques Appliquées, École PolytechNique, 91128, Palaiseau, France

E-mail address: santacesaria@cmap.polytechnique.fr 